\title{
Memory of Chirality in the Transannular Cyclization of Cyclodecenyl Radicals
}

\author{
Jackline E. Dalgard and Scott D. Rychnovsky* \\ Department of Chemistry, 516 Rowland Hall, University of \\ California, Irvine, CA 92697.
}

\section{Experimental Procedures and Compound Characterization}

\section{General Experiment Details}

All moisture-and air-sensitive reactions were carried out in flame- or oven-dried glassware using magnetic stirring under a positive pressure of argon gas. Standard syringe/septa techniques were employed. Reaction solvents were distilled or obtained from an alumina filtration system when necessary. Thin layer chromatography was performed on Whatman silica gel PE SIL G/UV plates. Concentration of organic solutions was performed using a Büchi rotary evaporator. Flash chromatography was performed on EM Science 230-400 mesh silica gel. Infrared spectra were recorded on a MIDAC Grams/Prospect FT-IR. NMR spectra were recorded on Brüker GN 500, Brüker Omega 500, and Brüker DRX 400 MHz FTNMR instruments. Proton NMR spectra were obtained using $\mathrm{CDCl}_{3}$ as solvent and referenced to residual protiated solvent ( $\left.\delta 7.26 \mathrm{ppm}\right)$ or $\mathrm{C}_{6} \mathrm{D}_{6}$ and referenced to $\delta 7.16 \mathrm{ppm}$. Carbon NMR spectra were recorded in ppm relative to the residual solvent signal: $\mathrm{CDCl}_{3}(\delta 77.0 \mathrm{ppm})$ or $\mathrm{C}_{6} \mathrm{D}_{6}(\delta 128.4 \mathrm{ppm})$. Mass spectra were determined on an AE2-MS 30, a PG 7070E-HF, a CG Analytical 7070E, or a Fisions autospec spectrometer. Optical rotations were measured with a Jasco DIP-370 digital polarimeter. Tetrahydrofuran, ethyl ether and methylene chloride were dried by 
filtration through alumina according to the procedure by Grubbs. ${ }^{1}$ Capillary GC analysis was performed on a Hewlett Packard Model 6890 instrument equipped with a FID detector. All reagents were purchased from Aldrich Chemical Co. or Acros and were used as received, unless otherwise stated. Elemental analyses were performed by M-HW Laboratories (Phoenix, AZ).

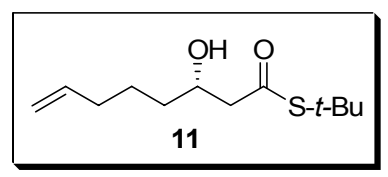

$\beta$-Hydroxy thioester 11. A mixture of $R$-(+)-1,1'-Bi-2-napthol (793 mg, $2.77 \mathrm{mmol}, 0.2$ equiv), $\mathrm{Ti}(\mathrm{O}-i-\mathrm{Pr})_{4}(0.82 \mathrm{~mL}, 2.77 \mathrm{mmol}, 0.2$ equiv) and oven dried powdered $4 \AA$ molecular sieves $(5.7 \mathrm{~g})$ in anhydrous ether $(57 \mathrm{~mL})$ was heated at reflux for $1 \mathrm{~h}$. The blood-red mixture was cooled to room temperature and 5-hexenal $(1.4 \mathrm{~g}, 14.3 \mathrm{mmol}, 1.0$ equiv) was added. After 5 min of stirring, the resultant solution was cooled to $-78{ }^{\circ} \mathrm{C}$ and 1-tert-butylthio-1-(trimethylsilyl)oxy ethene (4.6 mL, $20.0 \mathrm{mmol}, 1.4$ equiv) was added. The mixture was warmed to $-25^{\circ} \mathrm{C}$ and stirred $20 \mathrm{~h}$ then $\mathrm{pH} 7$ phosphate buffer was added and the contents were stirred for $15 \mathrm{~min}$. The resultant solution was filtered through a pad of Celite and the filtrate was extracted with ethyl ether $(2 \times 30 \mathrm{~mL})$, the combined extracts washed with brine and dried $\left(\mathrm{MgSO}_{4}\right)$. The ethereal layers were concentrated in vacuo to give the unpurified product as the free alcohol and silylated alcohol, which was treated with $10 \% \mathrm{HCl} / \mathrm{MeOH}(100 \mathrm{~mL})$. After $3 \mathrm{~h}$, the solution was concentrated in vacuo and the resultant oil was purified by column chromatography

\footnotetext{
1 Pangborn, A. B.; Giardello, M. A.; Grubbs, R. H.; Rosen, R. K.; Timmers, F. J. Organometallics
} 1996, 15, 1518-1520. 
$\left(\mathrm{SiO}_{2}, 30 \%\right.$ ether/hexanes, collected $\left.\mathrm{R}_{\mathrm{f}} 0.42\right)$ to give $3.29 \mathrm{~g}(89 \%, 89 \%$ ee, determined by Mosher ester analysis) of $\mathbf{1 1}$ as a colorless oil: $[\alpha]^{22}{ }_{\mathrm{D}}+20.1^{\circ}\left(c \mathrm{0.66}, \mathrm{CHCl}_{3}\right)$; IR (neat) $3434,3077,1681,1364,1163 \mathrm{~cm}^{-1} ;{ }^{1} \mathrm{H}$ NMR $\left(500 \mathrm{MHz}, \mathrm{CDCl}_{3}\right) \delta 5.79(\mathrm{ddt}, J=17.1$, 10.3, $6.7 \mathrm{~Hz}, 1 \mathrm{H}), 5.00(\mathrm{dd}, J=17.1,1.9 \mathrm{~Hz}, 1 \mathrm{H}), 4.95(\mathrm{dd}, J=10.2,2.0 \mathrm{~Hz}, \mathrm{I} \mathrm{H}), 4.06-$ $3.99(\mathrm{~m}, 1 \mathrm{H}), 2.76(\mathrm{~d}, J=3.8 \mathrm{~Hz}, 1 \mathrm{H}), 2.54(\mathrm{dd}, J=15.7,3.1 \mathrm{~Hz}, 1 \mathrm{H}), 2.56(\mathrm{dd}, J=$ 15.7, $8.7 \mathrm{~Hz}, 1 \mathrm{H}), 2.08-2.06$ (m, $2 \mathrm{H}), 1.54-1.43$ (m, $4 \mathrm{H}), 1.47$ (s, $9 \mathrm{H}) ;{ }^{13} \mathrm{C}$ NMR (125 $\left.\mathrm{MHz}, \mathrm{CDCl}_{3}\right) \delta 200.6,138.5,114.7,68.6,50.9,48.5,35.9,33.5,29.8,24.6$; HRMS (FAB) calcd for $\left[\mathrm{C}_{12} \mathrm{H}_{22} \mathrm{O}_{2} \mathrm{~S}+\mathrm{NH}_{4}\right]^{+} 231.1419$, found 231.1422 .

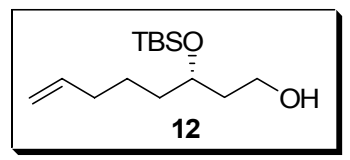

Alcohol 12. A flame-dried $100 \mathrm{~mL}$ round bottom flask was charged with $\beta$-hydroxy thioester 11 (3.24 g, $14.1 \mathrm{mmol}, 1.0$ equiv) in anhydrous $\mathrm{CH}_{2} \mathrm{Cl}_{2}(51 \mathrm{~mL})$. The solution was cooled to $0{ }^{\circ} \mathrm{C}$ and 2,6-lutidine $(2.1 \mathrm{~mL}, 18.3 \mathrm{mmol}, 1.3$ equiv) was added via syringe. The ice- $\mathrm{H}_{2} \mathrm{O}$ bath was removed and the reaction mixture was allowed to stir for $50 \mathrm{~min}$, after which an additional 0.3 equiv of 2,6-lutidine $(0.5 \mathrm{~mL}, 4.36 \mathrm{mmol})$ and tertbutyldimethylsilyltriflate $(4.2 \mathrm{~mL}, 18.3 \mathrm{mmol})$ were added via syringe. The resultant solution was stirred an additional $1 \mathrm{~h}$, after which the reaction mixture was diluted with an equal volume of ether and washed with satd. aq. $\mathrm{NaHCO}_{3}(1 \times 30 \mathrm{~mL})$, brine $(1 \times 30$ $\mathrm{mL}$ ), dried over anhydrous $\mathrm{MgSO}_{4}$ and concentrated in vacuo to give $4.45 \mathrm{~g}$ (quantitative yield) of the silyl ether as a colorless oil: $[\alpha]^{22}{ }_{\mathrm{D}}+28.6^{\circ}\left(c 1.02, \mathrm{CHCl}_{3}\right)$; IR (neat) 3078 , $1682,1364,1255,1078,836,775 \mathrm{~cm}^{-1} ;{ }^{1} \mathrm{H}$ NMR $\left(500 \mathrm{MHz}, \mathrm{CDCl}_{3}\right) \delta 5.78$ (ddt, $J=$ 
17.1, 10.3, $6.6 \mathrm{~Hz}, 1 \mathrm{H}), 5.00(\mathrm{dd}, J=17.1,1.8 \mathrm{~Hz}, 1 \mathrm{H}), 4.95(\mathrm{dd}, J=10.2,1.8 \mathrm{~Hz}, 1 \mathrm{H})$, 4.16-4.12 (m, $1 \mathrm{H}), 2.62(\mathrm{dd}, J=14.5,7.0 \mathrm{~Hz}, 1 \mathrm{H}), 2.51(\mathrm{dd}, J=14.5,5.6 \mathrm{~Hz}, 1 \mathrm{H})$, 2.04 (ddd, $J=7.0,7.0,7.0 \mathrm{~Hz}, 2 \mathrm{H}), 1.51-1.38$ (m, 4 H), 1.45 (s, 9 H), 0.86 (s, 9 H), 0.86 (s, $9 \mathrm{H}), 0.05$ (s, $3 \mathrm{H}), 0.05$ (s, $3 \mathrm{H}) ;{ }^{13} \mathrm{C}$ NMR $\left(125 \mathrm{MHz}, \mathrm{CDCl}_{3}\right) \delta$ 198.4, 138.6, 144.6, 69.1, 52.2, 48.0, 36.8, 33.6, 29.8, 25.8, 24.1, 18.0, -4.5, -4.7; HRMS (FAB) calcd for $\left[\mathrm{C}_{19} \mathrm{H}_{36} \mathrm{O}_{2} \mathrm{SSi}+\mathrm{H}\right]^{+}$345.2284, found 345.2283. Anal. calcd for $\mathrm{C}_{19} \mathrm{H}_{36} \mathrm{O}_{2} \mathrm{SSi}$ : C, 62.73; H, 10.53. Found: $\mathrm{C}, 62.88 ; \mathrm{H}, 10.28$. To a cooled $\left(-25^{\circ} \mathrm{C}\right)$ solution of the above silyl ether (1.96 g, 5.69 mmol, 1.0 equiv) in anhydrous $\mathrm{CH}_{2} \mathrm{Cl}_{2}(81 \mathrm{~mL})$ was added DIBALH (17.1 $\mathrm{mL}, 1.0 \mathrm{M}$ in toluene, $17.1 \mathrm{mmol}, 3.0$ equiv) slowly via syringe. The solution was stirred at $-30{ }^{\circ} \mathrm{C}$ for $2 \mathrm{~h}$. After quenching with Rochelle's salt $(70 \mathrm{~mL})$ and satd. aq. $\mathrm{NH}_{4} \mathrm{Cl}(7$ $\mathrm{mL})$, the aqueous layer was extracted with EtOAc $(3 \times 100 \mathrm{~mL})$, washed with brine $(1 \times$ $100 \mathrm{~mL})$, dried $\left(\mathrm{MgSO}_{4}\right)$ and concentrated. Purification by column chromatography $\left(\mathrm{SiO}_{2}, 40 \%\right.$ EtOAc/hexanes, $\left.\mathrm{R}_{\mathrm{f}} 0.33\right)$ provided $1.37 \mathrm{~g}$ of a colorless oil $(93 \%):[\alpha]^{22}{ }_{\mathrm{D}}$ $+26.3^{\circ}\left(c 0.67, \mathrm{CHCl}_{3}\right)$; IR (neat) $3364,3078,1642,1255,1062,836,775 \mathrm{~cm}^{-1} ;{ }^{1} \mathrm{H}$ NMR $\left(500 \mathrm{MHz}, \mathrm{CDCl}_{3}\right) \delta 5.79(\mathrm{ddt}, J=17.1,10.3,6.6 \mathrm{~Hz}, 1 \mathrm{H}), 5.03(\mathrm{dd}, J=17.1,1.7 \mathrm{~Hz}, 1$ H), 4.96 (dd, $J=10.2,1.7 \mathrm{~Hz}, 1 \mathrm{H}), 3.95-3.91(\mathrm{~m}, 1 \mathrm{H}), 3.86-3.83(\mathrm{~m}, 1 \mathrm{H}), 3.73-3.69$ (m, $1 \mathrm{H}), 2.45$ (br s, $1 \mathrm{H}), 2.08-2.03(\mathrm{~m}, 2 \mathrm{H}), 1.86-1.78(\mathrm{~m}, 1 \mathrm{H}), 1.69-1.51(\mathrm{~m}, 3 \mathrm{H})$, 1.43-1.37 (m, $2 \mathrm{H}), 0.90$ (s, $9 \mathrm{H}), 0.09$ (s, $3 \mathrm{H}), 0.07$ (s, $3 \mathrm{H}), 0.07$ (s, $3 \mathrm{H}) ;{ }^{13} \mathrm{C}$ NMR $\left(125 \mathrm{MHz}, \mathrm{CDCl}_{3}\right) \delta 138.6,114.6,71.7,60.2,37.7,36.2,33.7,36.2,33.7,25.8,24.6$, 18.0, -4.4, -4.7; HRMS (FAB) calcd for $\left[\mathrm{C}_{14} \mathrm{H}_{30} \mathrm{O}_{2} \mathrm{Si}+\mathrm{H}\right]^{+}$259.2094, found 259.2093. Anal. calcd for $\mathrm{C}_{14} \mathrm{H}_{30} \mathrm{O}_{2} \mathrm{Si}: \mathrm{C}, 65.06 ; \mathrm{H}, 11.70$. Found: C, 65.24; H, 11.53 . 


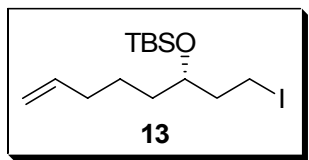

Iodide 13. To a cooled $\left(0{ }^{\circ} \mathrm{C}\right)$ solution of alcohol $12(332 \mathrm{mg}, 1.28 \mathrm{mmol}, 1.0$ equiv), triphenylphosphine (442 mg, $1.67 \mathrm{mmol}, 1.3$ equiv) and imidazole (139 mg, $2.05 \mathrm{mmol}$, 1.6 equiv) in $18 \mathrm{~mL} \mathrm{CH} \mathrm{Cl}_{2}$ was added $\mathrm{I}_{2}(424 \mathrm{mg}, 1.67 \mathrm{mmol}, 1.3$ equiv). The clear solution immediately turned yellow and the solution was stirred at $0{ }^{\circ} \mathrm{C}$ for $2 \mathrm{~h}$. Purified without workup $\left(\mathrm{SiO}_{2}, 10 \%\right.$ diethyl ether/hexanes, $\left.\mathrm{R}_{\mathrm{f}} 0.78\right)$. Obtained $408 \mathrm{mg}(94 \%)$ of 13 as a faint yellow oil: $[\alpha]^{22}+27.7^{\circ}\left(c 0.70, \mathrm{CHCl}_{3}\right)$; IR (neat) $3077,1641,1255,1069$, 836, $775 \mathrm{~cm}^{-1} ;{ }^{1} \mathrm{H}$ NMR $\left(500 \mathrm{MHz}, \mathrm{CDCl}_{3}\right) \delta 5.79(\mathrm{ddt}, J=17.1,10.3,6.6 \mathrm{~Hz}, 1 \mathrm{H})$, $5.03(\mathrm{dd}, J=17.1,1.7 \mathrm{~Hz}, 1 \mathrm{H}), 4.96(\mathrm{dd}, J=10.2,1.7 \mathrm{~Hz}, 1 \mathrm{H}), 3.75-3.72(\mathrm{~m}, 1 \mathrm{H})$, 3.24-3.19 (m, 1 H), 2.05-2.04 (m, 2 H), 1.98-1.95 (m, 2 H), 1.48-1.39 (m, 5 H), 0.89 (s, $9 \mathrm{H}), 0.08$ (s, $3 \mathrm{H}), 0.07$ (s, $3 \mathrm{H}) ;{ }^{13} \mathrm{C} \mathrm{NMR}\left(125 \mathrm{MHz}, \mathrm{CDCl}_{3}\right) \delta$ 138.6, 114.7, 72.0, 40.9, $36.2,33.8,25.9,24.1,18.1,3.3,-4.3,-4.4$; HRMS (FAB) calcd for $\left[\mathrm{C}_{14} \mathrm{H}_{29} \mathrm{IOSi}-\mathrm{CH}_{3}\right]^{+}$ 353.0789, found 353.0799. Anal. calcd for $\mathrm{C}_{14} \mathrm{H}_{29} \mathrm{IOSi}$ : C, 45.65; H, 7.93. Found: $\mathrm{C}$, 45.78; H, 7.87.

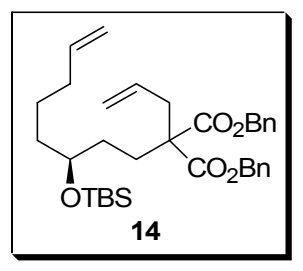

Diene 14. $\mathrm{NaH}$ (130 mg, $3.25 \mathrm{mmol}, 1.2$ equiv), washed $3 \times$ with hexanes, was suspended in $0.5 \mathrm{~mL}$ of THF. The suspension was cooled to $0{ }^{\circ} \mathrm{C}$ and allyl 
dibenzylmalonate ( $881 \mathrm{mg}, 2.71 \mathrm{mmol}, 1.0$ equiv), dissolved in $1.0 \mathrm{~mL}$ THF, was added via cannula, washing with $1.0 \mathrm{~mL}$ of THF. After $2 \mathrm{~h}$ of stirring at $0{ }^{\circ} \mathrm{C}$, iodide $\mathbf{1 3}$ was added via syringe and the solution was stirred, with warming to room temperature, for 13 h. The reagents were quenched with $5.0 \mathrm{~mL}$ satd. aq. $\mathrm{NH}_{4} \mathrm{Cl}$ and extracted with EtOAc $(3 \times 10 \mathrm{~mL})$. The combined extracts were washed with brine, dried over anhydrous $\mathrm{MgSO}_{4}$ and concentrated to provide and orange oil. Purification by silica gel chromatography (10\% ether/hexanes, $\mathrm{R}_{\mathrm{f}} 0.38$ in $30 \%$ EtOAc/hexanes) provided $982 \mathrm{mg}$ (94\%) of $\mathbf{1 4}$ as a colorless oil: $[\alpha]^{22}-4.3^{\circ}\left(c 1.17, \mathrm{CHCl}_{3}\right)$; IR (neat) $3070,3034,1734$, $1641,1457,1255,1209,835 \mathrm{~cm}^{-1} ;{ }^{1} \mathrm{H}$ NMR $\left(500 \mathrm{MHz}, \mathrm{CDCl}_{3}\right) \delta 7.32-7.28(\mathrm{~m}, 5 \mathrm{H})$, 7.27-7.24 (m, $5 \mathrm{H}), 5.76(\mathrm{ddd}, J=16.9,10.2,6.8,6.8 \mathrm{~Hz}, 1 \mathrm{H}), 5.63-5.54(\mathrm{~m}, 1 \mathrm{H})$, 5.18-4.92 (m, $8 \mathrm{H}), 3.59-3.53(\mathrm{~m}, 1 \mathrm{H}), 2.67(\mathrm{~d}, J=7.3 \mathrm{~Hz}, 2 \mathrm{H}), 2.05-1.96(\mathrm{~m}, 3 \mathrm{H})$, $1.88-1.82(\mathrm{~m}, 1 \mathrm{H}), 1.39-1.21(\mathrm{~m}, 6 \mathrm{H}), 0.86(\mathrm{~s}, 9 \mathrm{H}), 0.01(\mathrm{~s}, 3 \mathrm{H}), 0.00(\mathrm{~s}, 3 \mathrm{H}) ;{ }^{13} \mathrm{C}$ NMR (125 MHz, $\left.\mathrm{CDCl}_{3}\right) \delta 170.9,156.1,138.8,135.5,132.2,128.5,128.2,128.2,119.0$, 114.4, 97.3, 72.0, 66.9, 57.6, 37.0, 36.4, 33.8, 31.1, 28.4, 25.9, 24.4, 18.1, -4.5; HRMS $\left(\mathrm{CI}^{+}\right)$calcd for $\left[\mathrm{C}_{34} \mathrm{H}_{48} \mathrm{O}_{5} \mathrm{Si}+\mathrm{Na}\right]^{+}$587.3169, found 587.3152. Anal. calcd for $\mathrm{C}_{34} \mathrm{H}_{48} \mathrm{O}_{5} \mathrm{Si}: \mathrm{C}, 72.30 ; \mathrm{H}, 8.57$. Found: $\mathrm{C}, 72.10 ; \mathrm{H}, 8.36$.

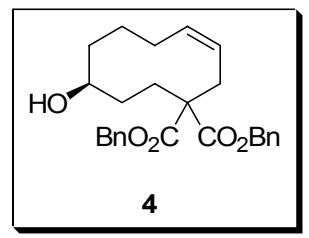

\section{Cyclodecenol 4.}


To a solution of diene $14\left(822 \mathrm{mg}, 1.46 \mathrm{mmol}, 1.0\right.$ equiv) in $1.6 \mathrm{~L}$ of degassed $\mathrm{CH}_{2} \mathrm{Cl}_{2}$ (0.001 $\mathrm{M}$ in substrate) was added 1,3-dimesityl-4,5-dihydroimimdazol-2-ylidene ruthenium catalyst (186 mg, $0.22 \mathrm{mmol}, 0.15$ equiv). The faint pink solution was heated at reflux for $18 \mathrm{~h}$. Additional catalyst ( $62 \mathrm{mg}, 73 \mu \mathrm{mol}, 0.5$ equiv) was added and the reaction mixture was heated for $6 \mathrm{~h}$. After cooling to room temperature, the reaction mixture was concentrated and poured through a plug of silica gel $(10 \%$ EtOAc/hexanes eluent) and purified by column chromatography ( $10 \%$ EtOAc/hexanes, $\left.R_{f} 0.29\right)$. Obtained $690 \mathrm{mg}(88 \%)$ of the macrocycle as a colorless oil: $[\alpha]^{22}{ }_{\mathrm{D}}-4.9^{\circ}(c$ 1.17, $\mathrm{CHCl}_{3}$ ); IR 3414, 2929, 1732, 1455, 1258, 1083, $697 \mathrm{~cm}^{-1} ;{ }^{1} \mathrm{H}$ NMR (500 MHz, $\mathrm{CDCl}_{3}$ ) $\delta$ 7.38-7.13 (m, $10 \mathrm{H}), 5.55-5.45(\mathrm{~m}, 1 \mathrm{H}), 5.26-5.00(\mathrm{~m}, 5 \mathrm{H}), 3.82-3.69(\mathrm{~m}, 1 \mathrm{H})$, 3.16-3.00 (m, $1 \mathrm{H}), 2.71-2.40(\mathrm{~m}, 2 \mathrm{H}), 2.15-1.20(\mathrm{~m}, 10 \mathrm{H}), 0.89(\mathrm{~s}, 3 \mathrm{H}), 0.86(\mathrm{~s}, 6 \mathrm{H})$, $0.00(\mathrm{~s}, 3 \mathrm{H}),-0.02(\mathrm{~s}, 3 \mathrm{H}) ;{ }^{13} \mathrm{C} \mathrm{NMR}\left(125 \mathrm{MHz}, \mathrm{CDCl}_{3}\right) \delta 171.4,171.1,170.9,170.8$, $135.7,135.5,134.8,133.5,128.6,128.5,128.3,128.2,128.1,128.1,128.0,127.9,126.5$, $126.2,125.9,124.0,123.7,77.3,76.8,72.0,71.9,70.8,66.9,57.1,56.4,30.9,30.2,29.7$, $29.5,29.3,28.6,27.9,26.8,26.4,25.8,25.1,24.3,23.8,20.5,18.1,18.0,-4.5,-4.9,-4.9$. HRMS $\left(\mathrm{CI}^{+}\right)$calcd for $\left[\mathrm{C}_{32} \mathrm{H}_{44} \mathrm{O}_{5} \mathrm{Si}+\mathrm{H}\right]^{+}$536.3036, found 537.3032. Anal. calcd for $\mathrm{C}_{32} \mathrm{H}_{44} \mathrm{O}_{5} \mathrm{Si}: \mathrm{C}, 71.60 ; \mathrm{H}, 8.26$. Found: $\mathrm{C}, 71.51 ; \mathrm{H}, 8.39$. The above silyl ether (197 mg, $0.37 \mathrm{mmol}, 1.0$ equiv) in $1.2 \mathrm{~mL}$ THF at $0{ }^{\circ} \mathrm{C}$ was treated with TBAF $(0.44 \mathrm{~mL}, 1.0 \mathrm{M}$ in THF, $0.44 \mathrm{mmol}, 1.2$ equiv). The reaction was warmed to room temperature and stirred $16 \mathrm{~h}$. The reagents were quenched with satd. aq. $\mathrm{NaHCO}_{3}(1 \mathrm{~mL})$ and extracted with EtOAc $(3 \times 5 \mathrm{~mL})$. The combined extracts were washed with brine, dried over anhydrous $\mathrm{MgSO}_{4}$ and concentrated. Purification by silica gel chromatography (30\% EtOAc/hexanes, $\left.\mathrm{R}_{\mathrm{f}} 0.24\right)$ provided $147 \mathrm{mg}(95 \%)$ of $\mathbf{4}$ as a colorless oil: IR (neat) 3416 , 
$1726,1498,1478,1471,1453,1263,1200,1163,1081 \mathrm{~cm}^{-1} ;{ }^{1} \mathrm{H}$ NMR $(500 \mathrm{MHz}$, $\left.\mathrm{CDCl}_{3}\right) \delta$ 7.33-7.28 (m, $\left.6 \mathrm{H}\right), 7.27-7.23(\mathrm{~m}, 4 \mathrm{H}), 5.59-5.45(\mathrm{~m}, 1 \mathrm{H}), 3.85-3.74(\mathrm{~m}, 1$ H), 3.14-2.98 (m, $1 \mathrm{H}), 2.63-2.37$ (m, $2 \mathrm{H}), 2.10-1.90$ (m, $3 \mathrm{H}), 1.85-1.32(\mathrm{~m}, 7 \mathrm{H}) ;{ }^{13} \mathrm{C}$ NMR $\left(125 \mathrm{MHz}, \mathrm{CDCl}_{3}\right) \delta 170.9,135.4,134.4,133.5,128.5,128.3,128.1,124.1,123.9$, $71.5,70.4,67.0,56.9,56.4,29.9,29.7,29.2,28.9,28.5,27.6,26.7,26.7,26.5,25.0,23.9$, 23.8, 20.3; HRMS $\left(\mathrm{CI}^{+}\right)$calcd for $\left[\mathrm{C}_{26} \mathrm{H}_{30} \mathrm{O}_{5}\right]^{+}$422.2093, found 422.2097 .

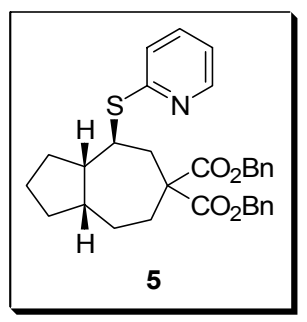

\section{Thioether 5.}

Representative procedure for radical deoxygenation: Oxalyl chloride $(62 \mu \mathrm{L}, 0.71 \mathrm{mmol}$, 20 equiv) was added via syringe to a solution of cyclodecenol 4 (15 mg, $36 \mu \mathrm{mol}, 1.0$ equiv) in $0.4 \mathrm{~mL}$ of $\mathrm{CH}_{2} \mathrm{Cl}_{2}$ at room temperature. After $3 \mathrm{~h}$, the solution was concentrated and the residue was redissolved in $3.6 \mathrm{~mL}$ of toluene. The reaction mixture was then cooled to $-15{ }^{\circ} \mathrm{C}$ and $N$-hydroxyl pyridine thione ( $6 \mathrm{mg}, 43 \mu \mathrm{mol}, 1.2$ equiv) and DMAP $(0.4 \mathrm{mg}, 4 \mu \mathrm{mol}, 0.1$ equiv) were added and the resultant solution was photolyzed for $1 \mathrm{~h}$. After an additional $1 \mathrm{~h}$ of stirring in the absence of light, the mixture was concentrated. Purification by column chromatography $\left(\mathrm{SiO}_{2}, \quad 20 \rightarrow 30 \%\right.$ EtOAc/hexanes, $\left.\mathrm{R}_{\mathrm{f}} 0.53\right)$ provided $9.3 \mathrm{mg}(51 \%, 84: 16 \mathrm{er}$, determined by chiral HPLC, OD-H column, $90: 10$ hexanes/IPA, $0.9 \mathrm{~mL} / \mathrm{min}$ ) of thioether 5 as a colorless oil: $[\alpha]^{22}$ 
$+32.6^{\circ}\left(c\right.$ 0.135, $\left.\mathrm{CHCl}_{3}\right)$; IR 3414, 2925, 1729, 1452, 1261, $1112 \mathrm{~cm}^{-1} ;{ }^{1} \mathrm{H}$ NMR (400 $\left.\mathrm{MHz}, \mathrm{CDCl}_{3}\right) \delta 8.38(\mathrm{~d}, J=4.0 \mathrm{~Hz}, 1 \mathrm{H}), 7.43(\mathrm{t}, J=7.3 \mathrm{~Hz}, 1 \mathrm{H}), 7.33-7.18(\mathrm{~m}, 8 \mathrm{H})$, 7.16-7.09 (m, $4 \mathrm{H}), 6.90$ (t, $J=5.3 \mathrm{~Hz}, 1 \mathrm{H}), 5.22$ (d, $J=12.4 \mathrm{~Hz}, 1 \mathrm{H}), 4.86(\mathrm{~d}, J=12.5$ $\mathrm{Hz}, 1 \mathrm{H}), 3.96(\mathrm{t}, J=11.0 \mathrm{~Hz}, 1 \mathrm{H}), 2.86(\mathrm{~d}, J=14.8 \mathrm{~Hz}, 1 \mathrm{H}), 2.55(\mathrm{dd}, J=14.2,7.2 \mathrm{~Hz}$, $1 \mathrm{H}), 2.49$ (dd, $J=14.8,10.6 \mathrm{~Hz}, 1 \mathrm{H}), 2.31-2.21(\mathrm{~m}, 1 \mathrm{H}), 2.17-2.05(\mathrm{~m}, 1 \mathrm{H}), 2.02-$ 1.90 (m, $2 \mathrm{H}), 1.90-1.78(\mathrm{~m}, 1 \mathrm{H}), 1.68-1.50(\mathrm{~m}, 4 \mathrm{H}), 1.44-1.22(\mathrm{~m}, 2 \mathrm{H}) ;{ }^{13} \mathrm{C}$ NMR $\left(125 \mathrm{MHz}, \mathrm{CDCl}_{3}\right) \delta 171.5,171.0,135.5,135.4,128.4,128.3,128.1,128.0,127.9,127.8$ 67.0, 66.9, 58.0, 49.4, 42.5, 41.8, 35.8, 34.9, 33.6, 28.4, 25.1; $\mathrm{HRMS}\left(\mathrm{CI}^{+}\right)$calcd for $\left[\mathrm{C}_{31} \mathrm{H}_{33} \mathrm{NO}_{4} \mathrm{~S}+\mathrm{H}\right]^{+}$516.2209, found 516.2185. 